\title{
miR-511 induces the apoptosis of radioresistant lung adenocarcinoma cells by triggering BAX
}

\author{
HAN-HAN ZHANG ${ }^{1 *}$, MIN PANG $^{1 *}$, WEI DONG ${ }^{2,3}$, JIA-XUAN XIN $^{1}$, YOU-JIE LI ${ }^{1}$, ZI-CHENG ZHANG $^{2}$, \\ LIANG YU ${ }^{4}$, PING-YU WANG ${ }^{1}$, BAO-SHENG LI $^{3}$ and SHU-YANG XIE ${ }^{1}$ \\ ${ }^{1}$ Key Laboratory of Tumour Molecular Biology, Department of Biochemistry and Molecular Biology, Binzhou \\ Medical University, Yantai, Shandong 264003; ${ }^{2}$ Department of Radiation Oncology, Cancer Hospital, \\ Tianjin Medical University, Tianjin 300060; ${ }^{3}$ Department of Radiation Oncology, Shandong Cancer Hospital, \\ Shandong Academy of Medical Sciences, Jinan 250117; ${ }^{4}$ Department of Radiotherapy, The Affiliated Hospital \\ of Binzhou Medical University, Yantai, Shandong 264000, P.R. China
}

Received October 23, 2013; Accepted December 18, 2013

DOI: 10.3892/or.2014.2973

\begin{abstract}
Radioresistance is one of the main reasons for the failure of radiotherapy in lung cancer. The present study was conducted to identify the role of miR-511 in suppressing the growth of radioresistant lung adenocarcinoma cells. First, a radioresistant A549/R cell line was generated after prolonged exposure to X-rays for $68 \mathrm{~Gy}$ ( $2 \mathrm{~Gy} / \mathrm{day}, 5$ days/week) and the radioresistance was confirmed by wound healing assay. Next, oncogenic TRIB2 was found to be upregulated in the radioresistant A549/R cells when compared to that of the control A549 cells as determined by western blot analysis. As the upstream miRNA, quantitative PCR showed that miR-511 expression was decreased in the radioresistant A549/R cells. Overexpression of miR-511 in miR-511-transfected A549/R cells inhibited cell growth and increased the number of apoptotic cells when compared with the control treatment. Flow
\end{abstract}

Correspondence to: Dr Shu-Yang Xie, Key Laboratory of Tumour Molecular Biology, Department of Biochemistry and Molecular Biology, Binzhou Medical University, No. 346 Guan Hai Road, Lai Shan, YanTai, Shandong 264003, P.R. China

E-mail: shuyangxie@aliyun.com

Dr Bao-Sheng Li, Department of Radiation Oncology, Shandong Cancer Hospital, Shandong Academy of Medical Sciences, Jinan 250117, P.R. China

E-mail: baoshli@yahoo.com

*Contributed equally

Abbreviations: NSCLC, non-small cell lung cancer; EGFR, epidermal growth factor receptor; miRNA, microRNA; MAPK, mitogen-activated protein kinase; MTT, 3-(4,5-dimethylthiazol-2yl)-2,5-diphenyltetrazolium bromide; DMSO, dimethyl sulfoxide; FACS, flow cytometry

Key words: lung adenocarcinoma, radioresistance, miR-511, BAX, TRIB2 cytometric analysis further demonstrated that the growth suppressive effect of miR-511 on A549/R cells was mediated by regulation of the cell cycle, most likely due to a block in the G1-S transition. Finally, our results showed that the expression of BAX was lower in the radioresistant A549/R cells when compared with that in the control A549 cells. After downregulation of TRIB2 by miR-511 treatment, BAX expression was obviously increased in the miR-511-transfected apoptotic A549/R cells when compared to that in the NC-treated or control cultures. In summary, our results revealed that miR-511 regulates the growth of radioresistant A549/R cells by increasing BAX expression through TRIB2, which suggests that miR-511 may be a potential therapeutic molecule for the treatment of radioresistant lung adenocarcinoma.

\section{Introduction}

Lung cancer is a disease characterized by uncontrolled cell growth in lung tissues. Due to the high incidence and mortality rate, lung cancer was the leading cause of cancer-related deaths in men and the second leading cause of cancer-related deaths in women $(1,2)$. Lung cancer is responsible for more than one million deaths worldwide annually. Among all lung cancer types, $75-85 \%$ of cases are non-small-cell lung cancer (NSCLC), including lung adenocarcinoma. Early diagnosis of NSCLC is difficult, and most patients are diagnosed at the middle or advanced stage. Thus, most patients have no opportunity for surgical resection. Therefore, these patients receive radiotherapy as a sole choice. Tyldesley et al showed that $\sim 60 \%$ of NSCLC cases require radiotherapy, both at initial treatment and at the later course of the disease (3). Postoperative radiotherapy alone, or intraoperative brachytherapy combined with preoperative radiation therapy and surgery were reported as the treatment modalities of superior sulcus tumors. Radiotherapy combined with chemotherapy is the best option for patients with stage III NSCLC (4).

Although radiotherapy plays a critical role in the management of NSCLC (5), tumor radioresistance, such as intrinsic radioresistance or acquired radioresistance, is one of the 
main obstacles for improving the efficacy of radiotherapy. Accumulating evidence indicates that radioresistance is related to certain genes, for example, p53 (6), epidermal growth factor receptor (EGFR) (7), DDB2, LOX and CDH2 (8). Overexpression of RAS leads to radioresistance, while ATM increases sensitivity to ionizing radiation (9). Investigation of the expression of these genes has increased our understanding of the molecular mechanisms and pathways involved in radioresistance, yet, the detailed functional mechanisms remain largely elusive.

microRNAs (miRNAs) are small non-coding RNAs 20-22 nucleotides long, which were originally characterized in eukaryotes as non-protein coding RNAs (10). The expression changes of miRNAs have subsequently been reported in many types of human tumors (11-13). As tumor-suppressors (miR-15a and miR-16-1) or oncogenes (miR-155 or members of the miR-17-92 cluster), miRNAs have been proposed to contribute to oncogenesis. The miRNA let-7 has also been demonstrated to regulate the radiation response of human cancers (14). Through suppressing let- $7 \mathrm{~g}$ processing, LIN28B plays an important role in the radiation response of lung cancer cells (15). These studies indicate that miRNAs regulate the radiation responses of various types of human cancers.

In a recent study, we found that miR-511 regulates lung adenocarcinoma A549 cell proliferation in vitro and in vivo (16). To further identify the role of miR-511 in radioresistant lung adenocarcinoma cells, we generated a radioresistant A549/R cell line and found that miR-511 regulated the growth of radioresistant A549/R cells by increasing BAX. This indicates that miR-511 is an important molecule for radioresistance therapy.

\section{Materials and methods}

Cell culture and transfection. Human lung adenocarcinoma A549 cells (Shanghai Institute of Cell Biology, China) were cultured in RPMI-1640 medium supplemented with $10 \%$ heatinactivated fetal calf serum (both from HyClone, USA) and $100 \mathrm{U} / \mathrm{ml}$ penicillin-streptomycin (Sigma, USA) at $37^{\circ} \mathrm{C}$ in $5 \%$ $\mathrm{CO}_{2}$.

All transfection experiments were carried out with Lipofectamine 2000 (Invitrogen, USA) according to the manufacturer's instructions. In brief, $5 \times 10^{5}$ cells were treated with $1 \mu \mathrm{g}$ miRNA and $2.5 \mu \mathrm{l}$ of Lipofectamine 2000. All transfections were carried out in triplicates.

Establishment of the radioresistant lung adenocarcinoma A549 cell line. A radioresistant lung adenocarcinoma A549 cell line was obtained after prolonged exposure to X-rays for 68 Gy (2 Gy/day, 5 days/week). Briefly, when cells grew to $\sim 60 \%$ confluence in $25 \mathrm{~cm}^{2}$ culture flasks, the A549 cells were irradiated with 2 Gy of X-ray irradiation with a linear accelerator (6-MV X-ray) at a rate of $3 \mathrm{~Gy} / \mathrm{min}$. One-centimeter thick tissue-equivalent bolus was placed on the top of the plate to ensure homogeneity. The fractionated irradiations were continued until the total concentration reached $68 \mathrm{~Gy}$. The radioresistant A549/R cell line was established.

Wound healing assay. Radioresistant A549/R cells were subjected to an in vitro wound healing assay with images captured at 0,24 and $48 \mathrm{~h}$ after incubation using a microscope. The rate of migration was measured by quantifying the distance that the A549/R cells moved from the edge of the scratch toward the center of the scratch (marked by imaginary dotted lines).

MTT assay. Forty-eight hours after transfection, the 3-(4,5-dimethylthiazol-2-yl)-2,5-diphenyltetrazolium bromide (MTT) assay was performed to detect the growth inhibition rate of A549/R cells. Cells $\left(1 \times 10^{4}\right)$ were cultured into each well of 96-well flat plates, and then $10 \mu 1 \mathrm{MTT}(5 \mathrm{mg} / \mathrm{ml})$ was added to each well. After $4 \mathrm{~h}$ the supernatant was removed and $100 \mu \mathrm{l}$ dimethyl sulfoxide (DMSO) (Sigma) was added. The OD value was measured with an enzyme-linked immunosorbent assay reader (ELx 800; USA) at $570 \mathrm{~nm}$. Cell growth inhibition rate $=\left(\mathrm{OD}_{\text {control }}-\mathrm{OD}_{\text {sample }}\right) / \mathrm{OD}_{\text {control }} \times 100(\%)(16,17)$.

Detection of apoptosis. The Annexin V-FITC/PI apoptosis detection kit (KeyGen Biotech, Nanjing, China) was used to stain the apoptotic cells according to the manufacturer's instructions. The radioresistant A549/R cells were gently washed with phosphate-buffered saline (PBS) twice. Then, the samples $\left(1 \times 10^{5}\right.$ cells) were centrifuged for $5 \mathrm{~min}$ at $2000 \mathrm{rpm}$, and the supernatant was discarded. Binding buffer (500 $\mu \mathrm{l})$ was added for resuspension. Then, $5 \mu \mathrm{l}$ Annexin V-FITC and $5 \mu \mathrm{l}$ propidium iodide were added to incubate the cells. The cells were then analyzed using a flow cytometer (FACS; Beckman Coulter, USA).

Analysis of cell cycle distribution. The radioresistant A549/R cells were harvested and washed with 1 X PBS buffer. Then, the cells were incubated in a $15-\mathrm{ml} \mathrm{V}$-bottomed tube on ice, and $3 \mathrm{ml}$ cold $\left(-20^{\circ} \mathrm{C}\right)$ absolute ethanol was added to fix the cells overnight. Propidium iodide staining solution $(1 \mathrm{ml})(50 \mu \mathrm{g} /$ $\mathrm{ml})$ was added to the cell pellets. Finally, $50 \mu \mathrm{l}$ of RNase A stock solution was added and the cell mixture was incubated for $3 \mathrm{~h}$ at $4^{\circ} \mathrm{C}$. The cell cycle distribution was detected by flow cytometry (Beckman Coulter).

Real-time PCR. miRNA from radioresistant A549/R cells was isolated by RNAiso (Takara, Shiga, Japan). Poly(A) was added using poly(A) polymerase (Ambion, Foster City, CA, USA). cDNA was synthesized and real-time PCR was performed to detect miR-511 levels as in our previous report (17). The forward primer used to detect miR-511 was 5'-GTGTCTTTTGCTCTGCAGTC-3' and the reverse primer was 5'-AACATGTACAGTCCATGGATG-3'. Human 5S rRNA served as the control. Forward primer of 5S rRNA was 5'-GCCATACCACCCTGAACG-3' and the reverse primer was 5'-AACATGTACAGTCCATGGATG-3'. SYBR ${ }^{\circledR}$ Premix Ex Taq $^{\text {TM }}$ kit (Takara, Shiga, Japan) was used according to the manufacturer's instructions. The expression of miR-511 was assessed using the RG3000 system (Corbett Research, Sydney, Australia) as follows: initiation with $3 \mathrm{~min}$ of denaturation at $95^{\circ} \mathrm{C}$, followed by 40 cycles of amplification with $20 \mathrm{sec}$ of denaturation at $95^{\circ} \mathrm{C}, 20 \mathrm{sec}$ at $56^{\circ} \mathrm{C}$ for annealing, and $20 \mathrm{sec}$ of extension at $72^{\circ} \mathrm{C}$. Fluorescence was detected at $585 \mathrm{~nm}$.

Western blotting. Cells were lysed in cold lysis buffer with $1 \mathrm{mM}$ phenylmethanesulfonyl fluoride for $30 \mathrm{~min}$ on ice. 

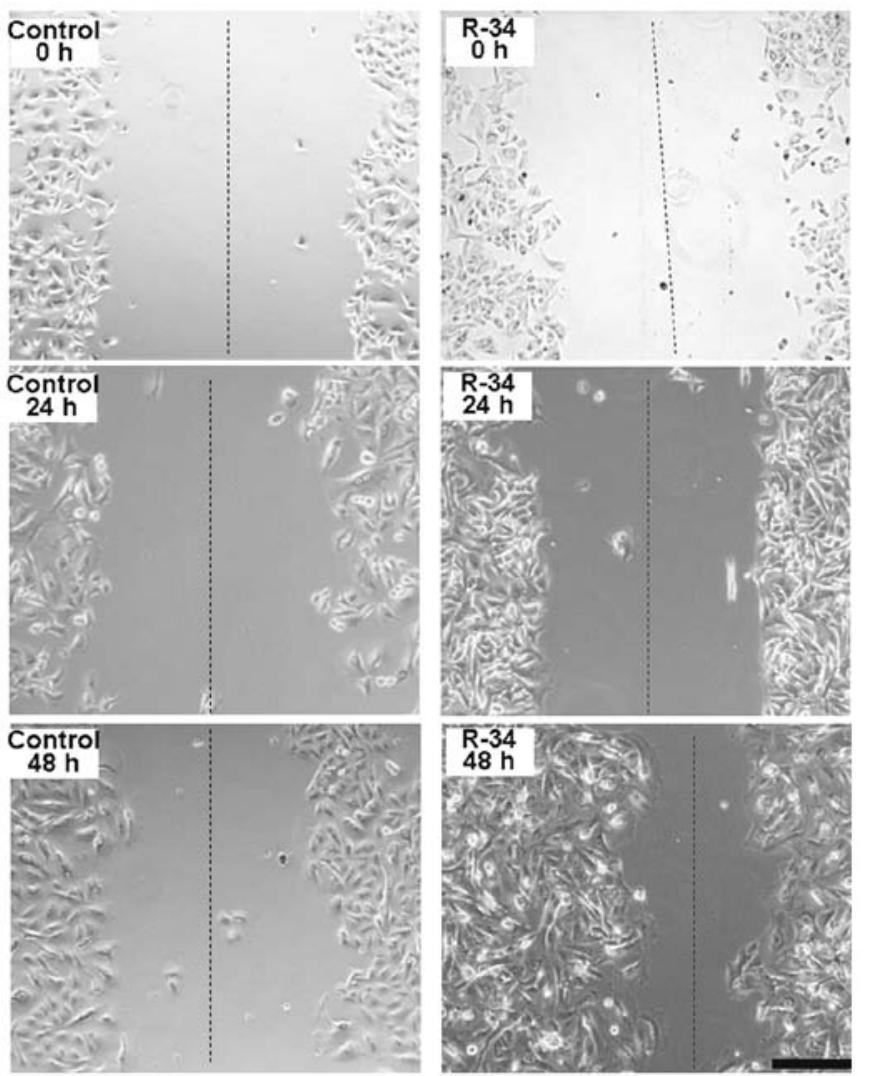

Figure 1. Wound healing assay. The cultures were subjected to an in vitro scratch assay with images captured at 0,24 and $48 \mathrm{~h}$ following incubation. A higher number of radioresistant A549/R cells were found to be alive and moved to the center of the scratch at 24 or $48 \mathrm{~h}$ when compared with that of A549 control cells. Control, A549 control cells. R-34, radioresistant A549/R cells exposed to X-rays for $68 \mathrm{~Gy}$. Line, the center of the scratch.

Protein concentrations were detected using Coomassie Blue Fast staining solution (Beyotime, China), and each $35 \mu \mathrm{g}$ protein sample was subjected to SDS-PAGE, and transferred to polyvinylidene difluoride membranes. After incubation in a blocking buffer (containing 10\% non-fat milk and $0.1 \%$ Tween-20) for $1 \mathrm{~h}$, the membranes were immunoblotted with antibodies against TRIB2 (1:500; Santa Cruz Biotechnology, USA) and BAX (1:500; BioWorld, USA). The membranes were stripped and re-blotted with actin antibody (1:500; Beijing Zhongshan Golden Bridge Technology Co., Ltd., China) as a control. Secondary antibodies conjugated to horseradish peroxidase were incubated for detection. Images were captured by the FluorChem FC2 gel imaging system (Alpha Innotech, USA).

Statistical analysis. The SAS software was used to analyze the significance of the results. The Student's t-test (or one-way ANOVA) was used for intergroup comparisons. A $\mathrm{P}<0.05$ was considered to indicate a statistically significant result.

\section{Results}

Generation of radioresistant A549/R cells. Radioresistance is one of the main reasons for the failure of radiotherapy in lung cancer (18). To study the mechanism of the radioresistance of lung adenocarcinoma, we established radioresistant A549/R
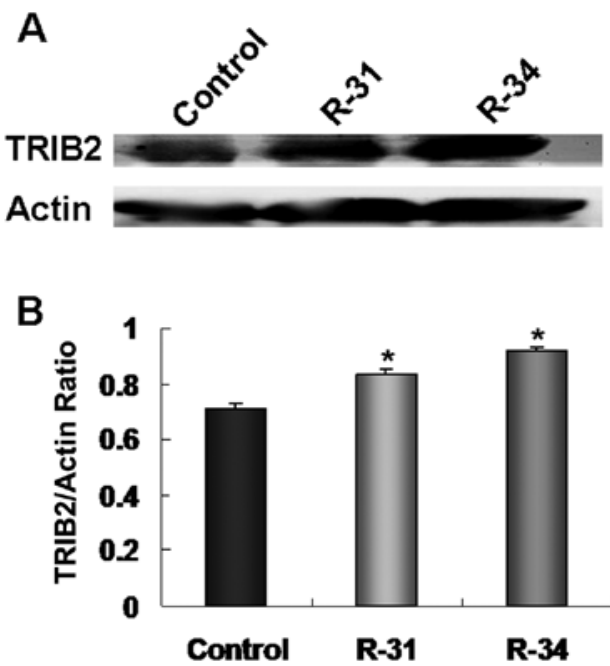

Figure 2. TRIB2 expression in radioresistant A549/R cells. (A) TRIB2 expression was increased in the A549/R cells when compared with that in the control A549 cells. (B) Relative values for the TRIB2/actin ratio in A. ${ }^{*} \mathrm{P}<0.05$ vs. the control A549 cells. Control, A549 control cells. R-31, A549/R cells exposed to 62 Gy of X-rays; R-34, radioresistant A549/R cells exposed to 68 Gy of X-rays.

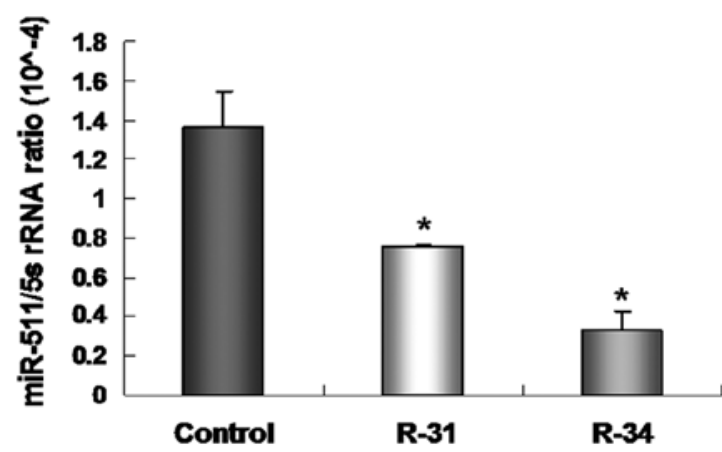

Figure 3. miR-511 expression in the radioresistant A549/R cells. Real-time PCR showed that miR-511 expression was obviously decreased in the A549/R cells when compared to that in the control A549 cells. ${ }^{*} \mathrm{P}<0.01$ vs. the control A549 cells.

cells after prolonged exposure to X-rays for $68 \mathrm{~Gy}$ ( 2 Gy/day, 5 days/week). The radioresistance of the A549/R cells was confirmed by a wound healing assay, which indicated that a higher number of cells were alive in the radioresistant A549/R cultures than those in the control A549 cells after exposure with $4 \mathrm{~Gy}$ of X-ray. Forty-eight hours later, more cells were found to move from the edge of the scratch toward the center of the scratch in the radioresistant A549/R cultures than the number in the control A549 cells (Fig. 1). MTT assay showed that a higher number of cells were alive in the radioresistant A549/R cultures than those in the control A549 cells $48 \mathrm{~h}$ after exposure to $4 \mathrm{~Gy}$ of $\mathrm{X}$-rays (data not shown).

TRIB2 is overexpressed in the radioresistant A549/R cells. In recent studies $(16,19)$, we found that TRIB2 plays an oncogenic role in the etiology of lung adenocarcinoma. To further investigate whether TRIB2 is involved in the radioresistance of lung adenocarcinoma, the expression of TRIB2 in the radioresistant A549/R cells was detected by western blotting. We found that 

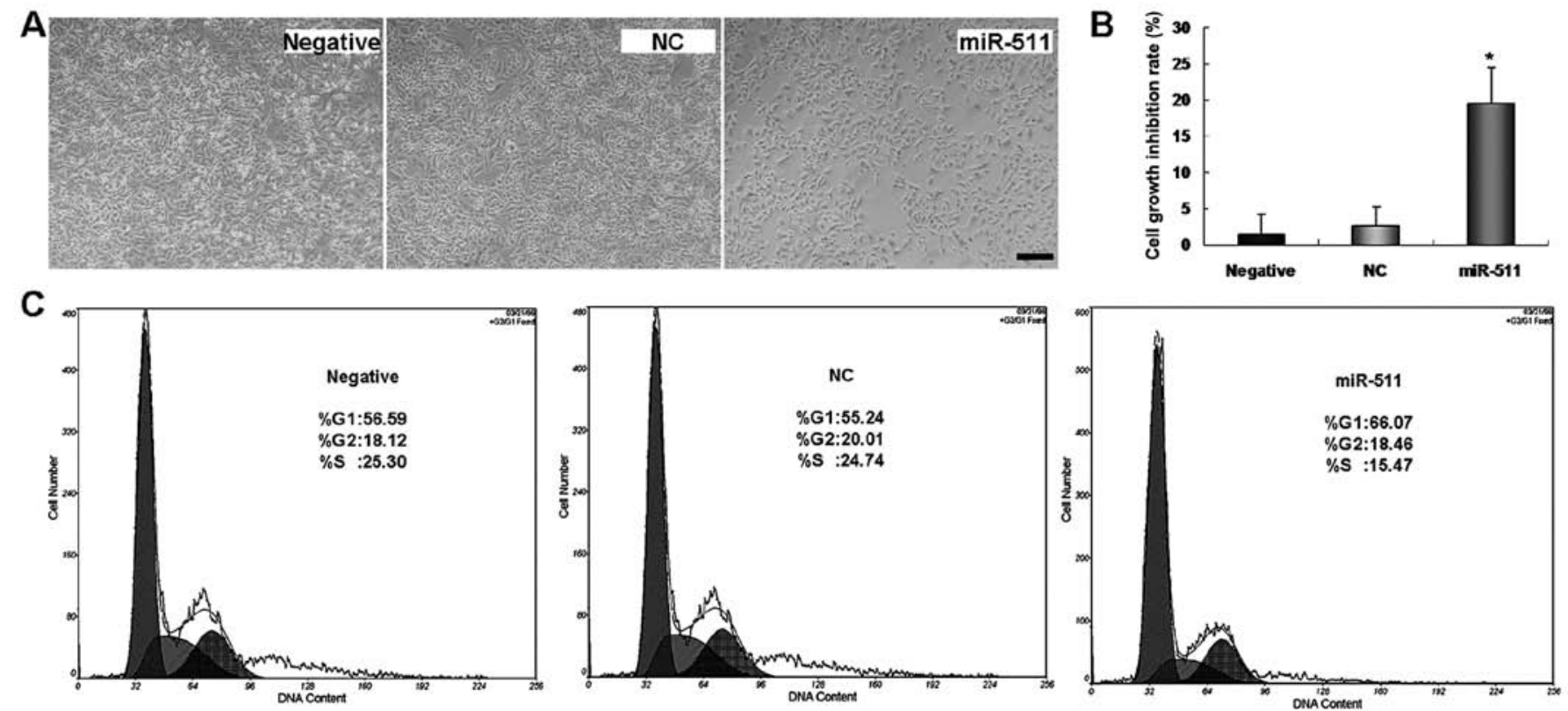

Figure 4. miR-511 suppresses A549/R cell proliferation. (A) Assessment of A549/R cell proliferation under an inverted microscope. A fewer number of live cells was noted in the miR-511-treated cells than that in the control cells. Scale bar, $100 \mu \mathrm{m}$. (B) MTT assay revealed that the growth inhibition rate was much higher in the miR-511-transfected A549/R cells than that in the control oligo-transfected (NC) cells. ${ }^{~} \mathrm{P}<0.01$ vs. untreated control or NC-transfected cells (C) Cell cycle detection. FACS results showed that the percentage of G1 phase cells was higher, but the percentage of S phase cells was lower in the miR511-transfected cells when compared with the control cultures. FACS, flow cytometry.
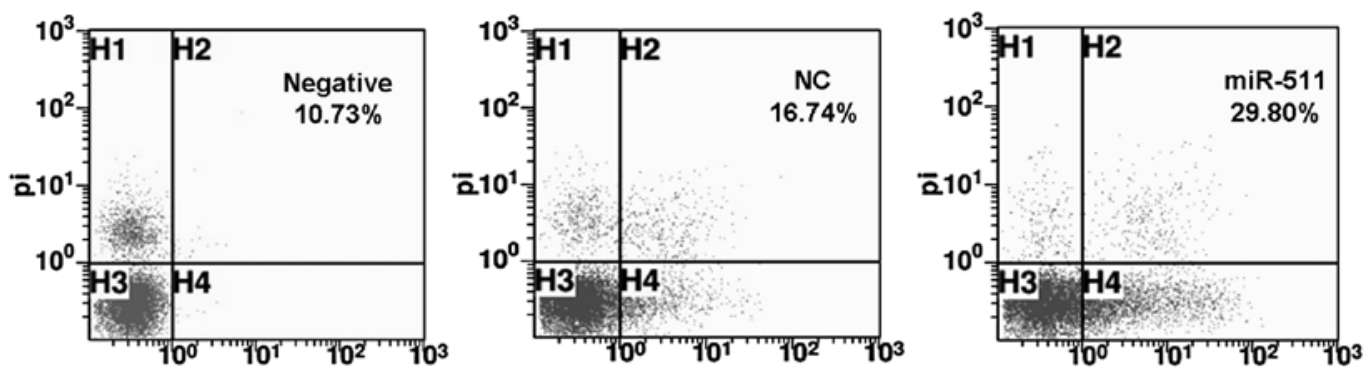

Figure 5. Analysis of apoptosis. FACS analysis revealed that the percentage of apoptotic A549/R cells was higher in the miR-511-transfected cells when compared with the control treatment. FACS, flow cytometry.

TRIB2 was expressed at a high level in the radioresistant A549/R cells (R-31 and R-34) when compared with this level in the control A549 cells (Fig. 2A and B), which indicates that TRIB2 plays an important role in the radioresistance of lung adenocarcinoma.

miR-511 inhibits the growth of radioresistant A549/R cells. Our previous study proved that miR-511, as a tumor-suppressor gene, suppresses A549 cell proliferation in vitro and in vivo by regulating TRIB2 (16). In the present study, we analyzed the levels of miR-511 in radioresistant A549/R cells by real-time PCR. The level of miR-511 was decreased in the radioresistant A549/R cells (R-31 and R-34) when compared with this level in the control A549 cells (Fig. 3).

When miR-511 was overexpressed in radioresistant A549/R cells, we found that miR-511 overexpression inhibited the growth of the radioresistant A549/R cells. Fewer miR-511-transfected A549/R cells were found to grow when compared with the NC or untreated cultures (Fig. 4A). MTT assay further proved the inhibitory role of miR-511 in the growth of radioresistant A549/R cells (Fig. 4B).

The above results indicate that miR-511 suppresses A549 cell proliferation. To further test whether the suppressive role of miR-511 is related to the regulation of the cell cycle, flow cytometric analysis was used to detect the changes in the cell cycle distribution after radioresistant A549/R cells were transfected with miR-511. A significant increase in the percentage of A549/R cells in the G1 phase (Fig. 4C) was found in the miR-511-treated A549/R cells when compared to this percentage in the control cultures, suggesting that the suppressive role of miR-511 is related to the regulation of the cell cycle, most likely due to a block in G1-S phase transition.

miR-511 induces A549/R cell apoptosis through downregulation of TRIB2. Next, we studied whether miR-511 induces A549/R cell apoptosis through FACS analysis. We found that the apoptotic rate was $29.80 \%$ in the miR-511-transfected A549/R cells, which was much higher than that in the control 

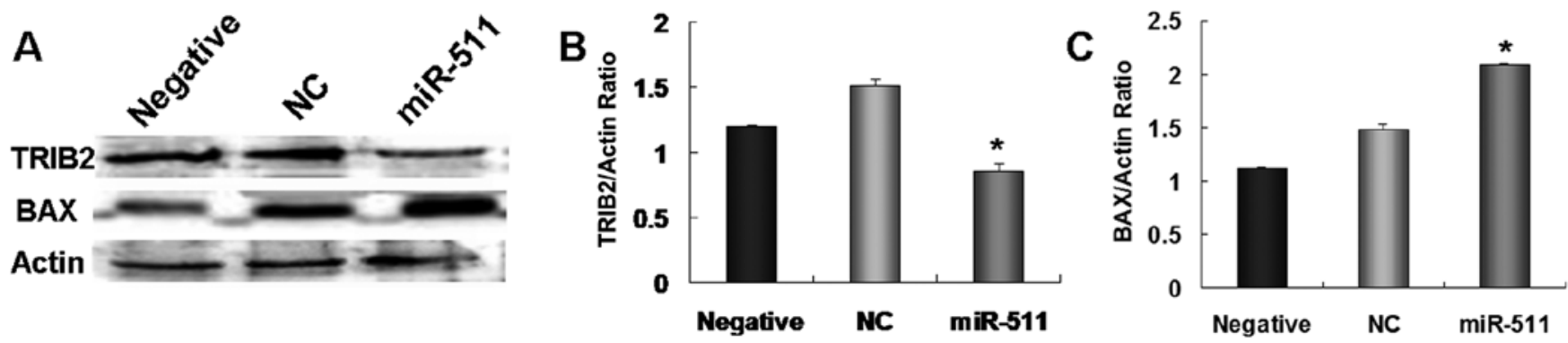

Figure 6. miR-511 regulates TRIB2 and BAX expression. (A) Western blotting revealed that TRIB2 expression was decreased, while BAX was increased in the miR-511-transfected A549/R cells when compared with the control treatment. (B) Relative values for the TRIB2/actin ratio in A. ${ }^{*} \mathrm{P}<0.05$ vs. the control treatment. (C) Relative values for BAX/actin ratio in A. ${ }^{*} \mathrm{P}<0.01$ vs. the control treatment.

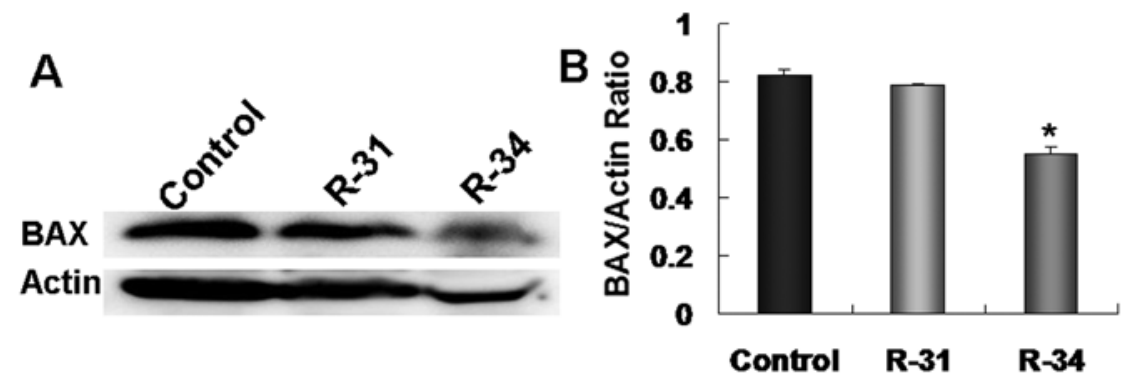

Figure 7. BAX expression in radioresistant A549/R cells. (A) BAX expression was reduced in the A549/R cells when compared with that in the control A549 cells. (B) Relative values for the BAX/actin ratio in A. "P<0.01 vs. the control A549 cells. Control, A549 control cells; R-31, A549/R cells exposed to 62 Gy; R-34, radioresistant A549/R exposed to 68 Gy.
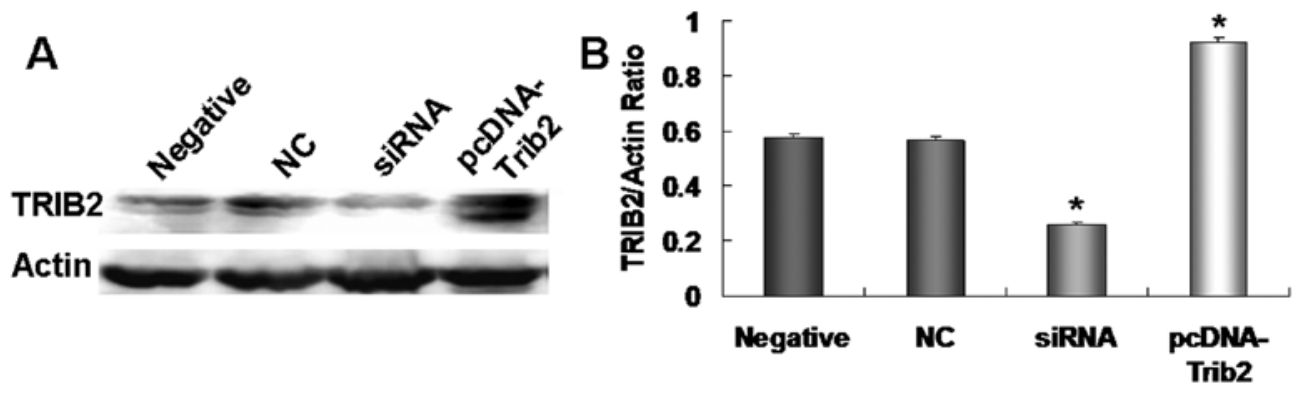

Figure 8. TRIB2 expression was detected after siRNA and pcDNA-Trib2 treatment. (A) Western blotting revealed that TRIB2 expression was decreased following siRNA treatment, while it was increased in the pcDNA-Trib2-transfected A549/R cells compared with the control cultures. (B) Relative values for the TRIB2/actin ratio in $\mathrm{A} .{ }^{*} \mathrm{P}<0.01$ vs. the control treatment.

NC-treated (16.74\%) or negative cultures (10.73\%). This suggests that miR-511 induces the apoptosis of radioresistant A549/R cells (Fig. 5).

The above results demonstrated that TRIB2 plays an important role in the radioresistance of lung adenocarcinoma. As an upstream miRNA, miR-511 may suppress A549 cell proliferation by regulating TRIB2 (16). Thus, we tested whether miR-511 regulates TRIB2 expression in radioresistant A549/R cells. Our results showed that the expression of TRIB2 was much lower in the miR-511-treated radioresistant A549/R cells than that in the control cultures (Fig. 6A and B). These results indicate that miR-511 also induces apoptosis in radioresistant A549/R cells by downregulation of TRIB2.

miR-511 increases BAX expression in apoptotic A549/R cells. TRIBs can regulate mitogen-activated protein kinase (MAPK) activation $(19,20)$, which further triggers BAX activation and translocation from the cytosol to the mitochondria (21). Therefore, we aimed to ascertain whether miR-511 induces radioresistant A549/R cell apoptosis by triggering BAX activation. We found that the expression of apoptotic gene Bax was lower in the radioresistant A549/R cells (R-31 and R-34) when compared with the expression levels in the control A549 cells (Fig. 7A and B). Nevertheless, after downregulation of TRIB2 by miR-511 treatment, BAX expression was obviously increased in the miR-511-transfected apoptotic A549/R cells when compared to that in the NC-treated and control cultures (Fig. 6A and C). Our results indicate that miR-511 induces A549/R cell apoptosis by increasing the BAX level through regulation of TRIB2.

To further test the regulatory role of miR-511 in increasing the BAX level through TRIB2, we designed an siRNA 
A

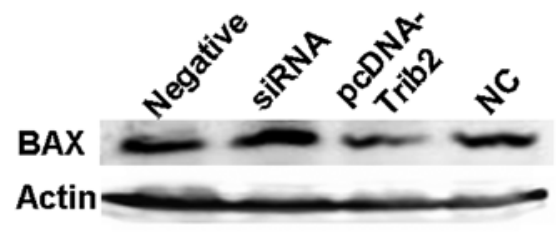

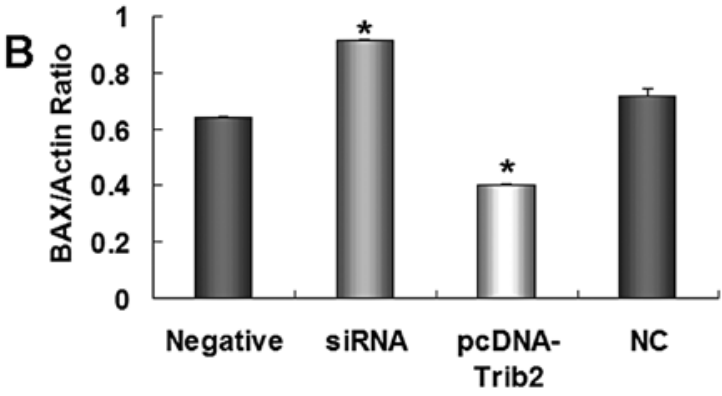

Figure 9. BAX expression was detected following siRNA and pcDNA-Trib2 treatment. (A) Western blotting revealed that BAX expression was enhanced following siRNA treatment, while BAX expression was decreased in the pcDNA-Trib2-transfected A549/R cells when compared with the control cultures. (B) Relative values for the $\mathrm{BAX} /$ actin ratio in $\mathrm{A} .{ }^{*} \mathrm{P}<0.01$ vs. the control treatment.

specific to TRIB2 to inhibit TRIB2 expression as a negative control and constructed a pcDNA-TRIB2 vector to increase TRIB2 protein as a positive control according to our previous studies $(16,19)$. When radioresistant A549/R cells were treated with the siRNA and pcDNA-TRIB2 vector, we found that siRNA specific to TRIB2 significantly inhibited TRIB2 expression, and TRIB2 was overexpressed in the pcDNA-TRIB2-treated cultures (Fig. 8A and B). After TRIB2 expression was decreased by siRNA, BAX expression was increased 1.5-fold compared with that in the NC or negative control cultures (Fig. 9A and B). When TRIB2 was upregulated, BAX expression was reduced obviously in the pcDNA-TRIB2treated A549/R cells when compared with those in the NC or negative control cultures (Fig. 9A and B). Our results suggest that miR-511 regulates the growth of radioresistant A549/R cells by overexpression of BAX through TRIB2.

\section{Discussion}

Radioresistance is one of the main obstacles to increasing the efficacy of radiotherapy for NSCLC. However, the nature of acquired radioresistance is still unclear (22). In the present study, we established a radioresistant A549/R cell line to investigate the role of miR-511 in suppressing the growth of radioresistant lung adenocarcinoma cells. Our results showed that overexpression of miR-511 inhibited the growth of A549/R cells and induced A549/R cell apoptosis. The suppressive role of miR-511 in regulating the growth of A549/R cells was most likely due to a block in the G1-S phase transition of the cell cycle. Our results further demonstrated that miR-511 regulates the growth of radioresistant A549/R cells by triggering BAX through TRIB2, which indicates the potential application of miR-511 for the therapy of radioresistant lung cancer.

Altered expression of miRNAs is commonly found in cancer and is associated with the pathogenesis of most malignancies, including lung cancer. miRNA expression directly affects cell proliferation, apoptosis and tumorigenesis $(23,24)$. miRNAs serve as either oncogenes or tumor-suppressor genes, depending on their targets. High miR-21 expression in tumors was demonstrated to be associated with poor survival in cancer patients, and plays a significant role in cancer growth by regulating stemness in cancer cells (25). In gastric cancer, upregulated miR-143 was found to be associated with gastric tumor size, stage, lymph node and distant metastases (26). Certain miRNAs can inhibit tumor growth.
For example, miR-34 is involved in the downstream p53 pathway, and is a potential tumor-suppressor in cancer stem cell self-renewal and survival (27). As tumor-suppressor genes, miR-511 and miR-1297 were found to suppress A549 cell proliferation in vitro and in vivo by suppressing TRIB2 and further increasing $\mathrm{C} / \mathrm{EBP} \alpha$ expression (16). Similarly, in the present study, we further demonstrated the role of miR-511 in suppressing the growth of radioresistant A549/R cells.

Radioresistance is one of the main obstacles to radiotherapy, and a number of miRNAs are involved in the radioresistance of tumors. For example, miR-21 is reported to mediate the resistance of glioblastoma cells against radiation via its target genes (PDCD4 and hMSH2) (28), indicating that miR-21 and its target genes may be used as potential molecular targets for clinical radiotherapy. Radiation may also upregulate miR-21 expression in mouse hippocampal cells through the EGFR/Stat3 pathway, and miR-21 activates the EGFR pathway (29). Yet, let-7a and let-7b were found to be downregulated following exposure to ionizing radiation. This decrease in expression was dependent on p53 and ATM. (30). Moreover, in the present study, we found that miR-511 expression was decreased in the radioresistant A549/R cells, but when miR-511 was overexpressed in A549/R cells, a higher number of apoptotic cells were detected in the miR-511-transfected A549/R cells when compared to the control cultures. Our results also revealed that the suppressive effect of miR-511 on the growth of A549/R cells was related to cell cycle regulation. These studies indicate that miRNAs play important roles in radioresistance.

TRIB2 is critical for both solid and non-solid malignancies. TRIB2 expression was found to be higher in acute lymphoblastic leukemia phenotypes when compared with acute myeloid leukemias, and is correlated with NOTCH1/ FBXW7 mutations (31). TRIB2 was also identified as a specific Wnt/ $\beta$-catenin signaling downstream target of liver cancer and is functionally important for liver cancer cell survival and transformation (32), indicating that TRIB2 functions as a signaling nexus to integrate the $\mathrm{Wnt} / \beta$-catenin, Hippo/YAP, and $\mathrm{C} / \mathrm{EBP} \alpha$ pathways in cancer cells. In our previous studies $(16,19)$, we found that TRIB2, as an oncogene, was targeted and negatively regulated by let-7c and miR-1297. Downregulation of TRIB2 expression resulted in lung cancer cell apoptosis. In the present study, we further demonstrated that TRIB2 was overexpressed in radioresistant lung cancer cells, which was negatively regulated by miR-511. 
TRIB2 can selectively modulate the activity of the p38 MAPK pathways $(19,33)$, which further triggers BAX expression (21). BAX expression was reported to be associated with radioresistance in cancer therapy. Overexpression of BAX and $c$-myc ensures the radiosensitivity of head and neck cancer (34), while knockdown of pro-apoptotic protein BAX resulted in an increase in lung cancer radiosensitivity in vitro (35). Similarly, we found that the expression of apoptotic BAX was decreased in the radioresistant A549/R cells. Following downregulation of TRIB2 by miR-511 treatment, the BAX expression was obviously increased in the radioresistant A549/R cells.

In summary, the present study showed that miR-511 regulates A549/R cell proliferation, which was associated with regulation of the cell cycle. miR-511 may also induce apoptosis of radioresistant A549/R cells by triggering BAX through TRIB2, indicating that miR-511 is a potential therapeutic molecule for the radiotherapy of cancer.

\section{Acknowledgements}

This study was supported by the NCET-10-0919, 'Taishan Scholar' position, National Natural Science Foundation (nos. 31371321 and 81200601), the Shandong Science and Technology Committee (no. ZR2009CL005), and the Foundation of Shandong Educational Committee of China (nos. J10LC60 and J11LC01).

\section{References}

1. Mokdad AH, Marks JS, Stroup DF and Gerberding JL: Actual causes of death in the United States, 2000. JAMA 291: 1238-1245, 2004.

2. Centers for Disease Control and Prevention: Tobacco use among adults - United States, 2005. MMWR Morb Mortal Wkly Rep 55: $1145-1148,2006$

3. Tyldesley S, Boyd C, Schulze K, Walker H and Mackillop WJ: Estimating the need for radiotherapy for lung cancer: an evidence-based, epidemiologic approach. Int J Radiat Oncol Biol Phys 49: 973-985, 2001.

4. Dillman RO, Herndon J, Seagren SL, Eaton WL Jr and Green MR: Improved survival in stage III non-small-cell lung cancer: sevenyear follow-up of cancer and leukemia group B (CALGB) 8433 trial. J Natl Cancer Inst 88: 1210-1215, 1996.

5. Bradley JD, Paulus R, Graham MV, et al: Phase II trial of postoperative adjuvant paclitaxel/carboplatin and thoracic radiotherapy in resected stage II and IIIA non-small-cell lung cancer: promising long-term results of the Radiation Therapy Oncology Group - RTOG 9705. J Clin Oncol 23: 3480-3487, 2005.

6. Biard DS, Martin M, Rhun YL, et al: Concomitant p53 gene mutation and increased radiosensitivity in rat lung embryo epithelial cells during neoplastic development. Cancer Res 54 3361-3364, 1994

7. Wang H, Yu JM, Yang GR, et al: Further characterization of the epidermal growth factor receptor ligand 11C-PD153035. Chin Med J 120: 960-964, 2007.

8. Xu QY, Gao Y, Liu Y, Yang WZ and Xu XY: Identification of differential gene expression profiles of radioresistant lung cancer cell line established by fractionated ionizing radiation in vitro. Chin Med J 121: 1830-1837, 2008.

9. Westphal CH, Rowan S, Schmaltz C, Elson A, Fisher DE and Leder P: atm and $p 53$ cooperate in apoptosis and suppression of tumorigenesis, but not in resistance to acute radiation toxicity. Nat Genet 16: 397-401, 1997.

10. Bartel DP: MicroRNAs: genomics, biogenesis, mechanism, and function. Cell 116: 281-297, 2004.

11. Calin GA and Croce CM: MicroRNA-cancer connection: the beginning of a new tale. Cancer Res 66: 7390-7394, 2006.

12. Calin GA, Garzon R, Cimmino A, Fabbri M and Croce CM: MicroRNAs and leukemias: how strong is the connection? Leuk Res 30: 653-655, 2006.
13. Hammond SM: MicroRNAs as oncogenes. Curr Opin Genet Dev 16: 4-9, 2006.

14. Weidhaas JB, Babar I, Nallur SM, et al: MicroRNAs as potential agents to alter resistance to cytotoxic anticancer therapy. Cancer Res 67: 11111-11116, 2007.

15. Jeong SH, Wu HG and Park WY: LIN28B confers radio-resistance through the posttranscriptional control of KRAS. Exp Mol Med 41: 912-918, 2009.

16. Zhang C, Chi YL, Wang PY, et al: miR-511 and miR-1297 inhibit human lung adenocarcinoma cell proliferation by targeting oncogene TRIB2. PLoS One 7: e46090, 2012.

17. Li YJ, Zhang YX, Wang PY, et al: Regression of A549 lung cancer tumors by anti-miR-150 vector. Oncol Rep 27: 129-134, 2012.

18. Yan L, Xu G, Qiao T, Chen W, Yuan S and Li X: CpG-ODN 7909 increases radiation sensitivity of radiation-resistant human lung adenocarcinoma cell line by overexpression of Toll-like receptor 9. Cancer Biother Radiopharm 28: 559-564, 2013.

19. Wang PY, Sun YX, Zhang S, et al: Let-7c inhibits A549 cell proliferation through oncogenic TRIB2 related factors. FEBS Lett 587: 2675-2681, 2013.

20. Kiss-Toth E, Bagstaff SM, Sung HY, et al: Human tribbles, a protein family controlling mitogen-activated protein kinase cascades. J Biol Chem 279: 42703-42708, 2004.

21. Lee JH, Lee SW, Choi SH, Kim SH, Kim WJ and Jung JY: p38 MAP kinase and ERK play an important role in nitric oxideinduced apoptosis of the mouse embryonic stem cells. Toxicol In Vitro 27: 492-498, 2013.

22. Ogawa K, Utsunomiya T, Mimori K, et al: Differential gene expression profiles of radioresistant pancreatic cancer cell lines established by fractionated irradiation. Int J Oncol 28: 705-713, 2006.

23. Volinia S, Calin GA, Liu CG, et al: A microRNA expression signature of human solid tumors defines cancer gene targets. Proc Natl Acad Sci USA 103: 2257-2261, 2006.

24. Lanza G, Ferracin M, Gafà R, et al: mRNA/microRNA gene expression profile in microsatellite unstable colorectal cancer. Mol Cancer 6: 54, 2007

25. Chung WM, Chang WC, Chen L, et al: MicroRNA-21 promotes the ovarian teratocarcinoma PA1 cell line by sustaining cancer stem/progenitor populations in vitro. Stem Cell Res Ther 4: 88, 2013.

26. Takagi T, Iio A, Nakagawa Y, Naoe T, Tanigawa N and Akao Y: Decreased expression of microRNA-143 and -145 in human gastric cancers. Oncology 77: 12-21, 2009.

27. Tarasov V, Jung P, Verdoodt B, et al: Differential regulation of microRNAs by p53 revealed by massively parallel sequencing: miR-34a is a p53 target that induces apoptosis and G1-arrest. Cell Cycle 6: 1586-1593, 2007.

28. Chao TF, Xiong HH, Liu W, Chen Y and Zhang JX: MiR-21 mediates the radiation resistance of glioblastoma cells by regulating PDCD4 and hMSH2. J Huazhong Univ Sci Technolog Med Sci 33: 525-529, 2013

29. Shi Y, Zhang X, Tang X, Wang P, Wang H and Wang Y: MiR-21 is continually elevated long-term in the brain after exposure to ionizing radiation. Radiat Res 177: 124-128, 2012.

30. Saleh AD, Savage JE, Cao L, et al: Cellular stress induced alterations in microRNA let-7a and let-7b expression are dependent on p53. PLoS One 6: e24429, 2011.

31. Hannon MM, Lohan F, Erbilgin Y, et al: Elevated TRIB2 with NOTCH1 activation in paediatric/adult T-ALL. Br J Haematol 158: 626-634, 2012.

32. Wang J, Park JS, Wei Y, et al: TRIB2 acts downstream of $\mathrm{Wnt} / \mathrm{TCF}$ in liver cancer cells to regulate YAP and C/EBP $\alpha$ function. Mol Cell 51: 211-225, 2013.

33. Wei SC, Rosenberg IM, Cao Z, Huett AS, Xavier RJ and Podolsky DK: Tribbles 2 (Trib2) is a novel regulator of toll-like receptor 5 signaling. Inflamm Bowel Dis 18: 877-888, 2012.

34. Csuka O, Remenár E, Koronczay K, Doleschall Z and Németh G: Predictive value of $\mathrm{p} 53, \mathrm{Bcl} 2$ and bax in the radiotherapy of head and neck cancer. Pathol Oncol Res 3: 204-210, 1997.

35. Kim KW, Mutter RW, Cao C, et al: Autophagy for cancer therapy through inhibition of pro-apoptotic proteins and mammalian target of rapamycin signaling. J Biol Chem 281: 36883-36890, 2006. 